\title{
Targeting of the Wnt/ $\beta$-Catenin Pathway in Chronic Lymphocytic Leukaemia may adversely affect CTLA-4 expression and function
}

\author{
Gary Lynch, Paul Kennedy, Stephen Bergin, Siobhan Glavey, Philip W Murphy, John Quinn, and Philip T Murphy* \\ Department of Haematology, Beaumont Hospital, Dublin, Ireland
}

${ }^{*}$ Correspondence to: Philip T Murphy, Department of Haematology, Beaumont Hospital, Dublin 9, Ireland, Tel: +353 1 8092622; Fax: +353 18093319 ; E-mail: philipmurphy@beaumont.ie

Received: February 02, 2018; Accepted: February 24, 2018; Published: February 28, 2018;

\begin{abstract}
In chronic lymphocytic leukemia, overexpression of CTLA-4 may be associated with a good outcome, whereas the Wnt/ $\beta$-catenin-regulated transcription factor LEF1 is a pro-survival factor and is markedly overexpressed compared to normal B cells. In this study, peripheral blood B cells from 20 patients with CLL were purified and a strong correlation between gene expression levels of CTLA- 4 and LEF- 1 was found. This suggests that CTLA-4 expression in CLL may be a target of $\mathrm{Wnt} / \beta$-catenin signalling.
\end{abstract}

Keywords: CLL; CTLA-4; Wnt/ß-catenin pathway; LEF1; CD38

\section{Highlights}

Percentage surface expression of CD38 and CTLA-4 and gene expression levels of CTLA-4, CCND1, LEF1 and STAT3 were measured in 20 patients with chronic lymphocytic leukemic. A strong positive correlation was found between gene expression levels of CTLA-4 and LEF-1.

Targeting of the Wnt/ $\beta$-catenin pathway in CLL may result in unwanted effects on CTLA-4 expression and function.

\section{Introduction}

Chronic lymphocytic leukemia (CLL) is a clonal proliferation of mature CD5+ CD19+ CD23+ B lymphocytes, characterized by progressive accumulation of leukemic cells in peripheral blood, bone marrow and lymphoid tissues. Cytotoxic T-lymphocyte-associated antigen 4 (CTLA-4, CD152) is a member of the CD28 receptor family and is mainly expressed on CD4+ T-cells. In CLL, overexpression of the CTLA-4 gene is associated with lower CD38-expression and, therefore, perhaps a better outcome [1]. CLL cells also exhibit aberrantly active Wnt signaling and Wnt/ $\beta$-catenin-regulated transcription factor lymphoid enhancer binding factor-1 (LEF1) has been shown to be a pro-survival factor in CLL [2]. In this study, we wished to further investigate the relationship between CD38 and CTLA-4 in CLL and their potential relationship with transcription factors LEF1, signal transducer and activator of transcription 3 (STAT3) and cyclin D1. In purified peripheral blood B cells from 20 patients with CLL, a strong positive correlation between gene expression levels of CTLA- 4 and LEF1 was found, suggesting that CTLA-4 expression in CLL may well be a target of Wnt $/ \beta$-catenin signalling.

\section{Material and methods}

After ethical approval and signed written consent, 20 patients with CLL (9 previously treated, 11 untreated) donated peripheral blood for this study. No patient had active therapy for CLL in the 3 months prior to blood donation. All patients had FISH analysis performed. CD19+ B lymphocytes were isolated using a magnetic bead separation technique (Invitrogen-Dynabeads). The percentage surface expression of CD38 and CTLA- 4 was measured by flow cytometry. Total RNA was isolated from the B cells by the RNeasy Mini Kit (QIAGEN). Gene expression levels of CTLA-4, cyclin D1 (CCND1), LEF1 and STAT3 were measured using RT-PCR (ABI 7500 Fast-Applied Biosystems). GAPDH was used as a reference gene. Statistical analyses of data were performed using Spearman rank correlation and Mann-Whitney U tests. Differences of $\mathrm{P}<0.05$ were considered statistically significant.

\section{Results}

Median (range) CD19+ B cell purity was 93.8 (84.8-98.5) \%, with CD $19+$ B cell purity $>90 \%$ in 19/20 cases. Median (interquartile range) percentage surface expression of CD38 and CTLA-4 was 8.36 (26.45) $\%$ and 43.32 (50.22) \% respectively. Median (range, interquartile range) $\triangle \mathrm{CT}$ gene expression levels of CCND1, CTLA-4, LEF1 and STAT3 were 11.89 (1.81), 4.79 (2.35), $4.82(0.89)$ and 9.12 (0.75) respectively. Gene expression of LEF1 showed significant positive correlations with gene expression levels of CTLA- 4 ( $r s=0.572, p=0.008)$, CCND1 $(\mathrm{rs}=0.61, \mathrm{p}=0.004)$ and STAT3 ( $\mathrm{rs}=0.587, \mathrm{p}=0.006)$. There was also a significant positive correlation between gene expression of CCND1 and of STAT3 ( $r s=0.486, p=0.03$ ). No significant correlations were 
found between percentage surface expression of CTLA-4 and gene expression levels of either CTLA-4 or of LEF1. Although we found a negative correlation between percentage surface expression of CTLA-4 and CD38, this was not statistically significant. Comparing untreated and previously treated patients or comparing patients with poor risk cytogenetics $(17 \mathrm{p}$ or $11 \mathrm{q}$ deletions: $\mathrm{n}=6)$ to those without, there was no significant difference in gene expression levels of CTLA4, CCND1, LEF1 and STAT3 or in surface expression of CTLA-4 and CD38.

\section{Discussion}

The Wnt signalling pathway has been shown to be activated in CLL cells and uncontrolled Wnt/ $\beta$-catenin signalling contributes to defective apoptosis in CLL [3]. Importantly, Wnt pathway activation leads to upregulation of $\beta$-catenin and subsequently LEF1 activation, which is markedly overexpressed in CLL compared to normal B cells [4] and appears to play an essential role in the leukaemogenesis of CLL [2]. Furthermore, cyclin D1, a downstream target of LEF-1, is overexpressed in CLL. Targeting of LEF-1 has been shown to induce apoptosis in CLL cells both in vitro and in vivo [5].

In CLL, CTLA-4 expression is higher on the leukemic cells that on their normal B cell counterparts. A recent study has shown that CTLA4 inhibits the proliferation/survival of CLL cells via regulation of the expression/activation of STAT1, NFATC2, Fos, Myc and Bcl-2 [6] and CTLA-4 blockade induces pro-survival signals in leukemic cells from CLL patients exhibiting high CTLA-4 expression [7]. However, CTLA-4 expression was also found to be the most highly induced gene following treatment with recombinant Wnt-3a in melanoma cell lines and CTLA-4 expression appeared to be directly regulated by the $\mathrm{Wnt} / \beta$-catenin pathway as the $\beta$-catenin responsiveness of CTLA-4 promoter region required a T-cell factor-1/LEF-1 consensus site [8]. In our study, CTLA-4 and LEF-1 gene expression levels were strongly correlated, suggesting that CTLA-4 expression in CLL may well also be a direct target of $\mathrm{Wnt} / \beta$-catenin signalling. Although the relationship between CTLA- 4 and the Wnt/ $\beta$-catenin pathway in CLL requires further study, the findings of this study suggest that targeting of the Wnt/ $\beta$-catenin pathway in CLL may result in unwanted effects on CTLA-4 expression and function.

This research did not receive any specific grant from funding agencies in the public, commercial, or not-for-profit sectors.

\section{Conflicts of interest: none}

\section{References}

1. Joshi AD, Hegde GV, Dickinson JD (2007) ATM, CTLA4, MNDA and HEM1 in high versus low CD38 expressing B-cell chronic lymphocytic leukemia. Clin Cancer Res 13: 5295-5304. [Crossref]

2. Gutierrez Jr A, Tschumper RC, Wu X (2010) LEF-1 is a prosurvival factor in chronic lymphocytic leukemia and is expressed in the preleukemic state of monoclonal B-cell lymphocytosis. Blood 116: 2975-2983. [Crossref]

3. Lu D, Zhao Y, Tawatao R (2004) Activation of the Wnt signaling pathway in chronic lymphocytic leukemia. Proc Natl Acad Sci USA. 101: 3118-3123. [Crossref]
4. Gandhirajan RK, Poll-Wolbeck SJ, Gehrke I, Kreuzer KA (2010) Wnt/B-catenin/ LEF-1 signaling in chronic lymphocytic leukemia (CLL): a target for current and potential therapeutic options. Curr Cancer Drug Targets 10: 716-727. [Crossref]

5. Gandhirajan RK, Staib PA, Minke K (2010) Small molecule inhibitors of Wnt/ $\beta$ carenin/Lef-1 signaling induces apoptosis in chronic lymphocytic leukemia cells in vitro and in vivo. Neoplasia 12: 326-335. [Crossref]

6. Mittal AK, Chaturvedi NK, Rohlfsen RA (2013) Role of CTLA4 in the proliferation and survival of chronic lymphocytic leukemia. PLoS ONE 8: e70352. [Crossref]

7. Ciszak L, Frydecka I, Wolowiec D, Szteblich A, Kosmaczewska A (2016) Patients with chronic lymphocytic leukemia (CLL) differ in the pattern of CTLA-4 expression on CLL cells: the possible implications for immunotherapy with CTLA4 blocking antibody. Tumour Biol 37: 4143-4157. [Crossref]

8. Shah KV, Chien AJ, Yee C, Moon RT (2008) CTLA-4 is a direct target of Wnt/ $\beta$ catenin signaling and is expressed in human melanoma tumors. J Invest Dermatol 128: 2870-2879. [Crossref]

\section{Citation:}

Gary Lynch, Paul Kennedy, Stephen Bergin, Siobhan Glavey, Philip W Murphy, John Quinn, and Philip T Murphy (2018) Targeting of the Wnt/ $\beta$-Catenin Pathway in Chronic Lymphocytic Leukaemia may adversely affect CTLA-4 expression and function. Internal Med Res Open J Volume 3(1): 1-2 